\title{
An Efficient Hybrid Method for 3D Scattering from Inhomogeneous Object Buried beneath a Dielectric Randomly Rough Surface
}

\author{
Hong-jie He, Li-xin Guo, and Wei Liu \\ School of Physics and Optoelectronic Engineering, Xidian University, Xian 710071, China \\ Correspondence should be addressed to Hong-jie He; hongjiewuli2009@163.com
}

Received 3 May 2017; Accepted 3 July 2017; Published 28 August 2017

Academic Editor: Pasquale Imperatore

Copyright @ 2017 Hong-jie He et al. This is an open access article distributed under the Creative Commons Attribution License, which permits unrestricted use, distribution, and reproduction in any medium, provided the original work is properly cited.

\begin{abstract}
An efficient iterative analytical-numerical method is proposed for three-dimensional (3D) electromagnetic scattering from an inhomogeneous object buried beneath a two-dimensional (2D) randomly dielectric rough surface. In the hybrid method, the electric and magnetic currents on the dielectric rough surface are obtained by current-based Kirchhoff approximation (KA), while the scattering from the inhomogeneous object is rigorously studied by finite element method (FEM) combined with the boundary integral method (BIM). The multiple interactions between the buried object and rough surface are taken into account by updating the electric and magnetic current densities on them. Several numerical simulations are considered to demonstrate the algorithm's ability to deal with the scattering from the inhomogeneous target buried beneath a dielectric rough surface, and the effectiveness of our proposed method is also illustrated.
\end{abstract}

\section{Introduction}

The scattering from an object buried beneath a randomly dielectric rough surface which can be modelled as sea surface and sands is a subject of great interest in many applications, for example, geophysical exploration, buried target detection, and remote sensing. Many researchers have paid much attention to this problem and many numerical methods have been proposed in the past decades. The method of moments (MoM) was applied to discuss the scattering from a rough surface with a two-dimensional (2D) target in $[1,2]$. The MoM with the dyadic Green's function of the background medium is applied in [3] to study the forward-scattering problem of three-dimensional (3D) objects buried under a $2 \mathrm{D}$ locally rough surface. To improve the computational efficiency and reduce the memory requirement, some asymptotic methods and hybrid methods are developed to study the scattering from a target buried beneath the rough surface. The cylindrical wave approach combined with the first-order small perturbation method (SPM) [4] was applied in [5] to study the scattering from a cylinder buried below a slightly rough surface. The SPM was applied to study the electromagnetic scattering from a 2D dielectric cylinder buried beneath a slightly rough surface [6]. The second-order small slope approximation was used in [7] to study the scattering from one dimensional (1D) sea surface. The fields scattered by an object below a rough surface were computed by the efficient propagation-inside-layer-expansion (PILE) method combined with the physical optics approximation [8]. Hybrid KA-BIM was proposed in [9] to study the scattering from a $2 \mathrm{D}$ target buried beneath a rough surface. An efficient hybrid KA-MoM was proposed to analyse the electromagnetic scattering from a 3D perfectly electric conducting (PEC) object buried beneath a dielectric rough surface [10]. However, these numerical methods and hybrid methods mentioned above, for both $2 \mathrm{D}$ and $3 \mathrm{D}$ scattering problems, are applicable to PEC or dielectric objects. They all suffer the inconvenience of dealing with the scattering from inhomogeneous objects buried beneath dielectric rough surface.

For general inhomogeneous objects, the volume integral equation method is suitable. A fast volume integral equation algorithm with dyadic Green's function [11] was 
developed to simulate the electromagnetic scattering from large inhomogeneous objects embedded in a planarly layered medium. The other appropriate choice for inhomogeneous objects is the finite element method (FEM) combined with the boundary integral method (BIM). The hybrid FEM/BIM method with the layered medium dyadic Green's function [12] was proposed to predict the scattering from inhomogeneous dielectric objects embedded in multilayered medium. But these methods cannot be applied to deal with the scattering from inhomogeneous object buried beneath randomly rough surface, since the analytical expression of the dyadic Green's function for the half space with rough surface can hardly be obtained. A hybrid FEM/MoM was applied in [13] to study the scattering from a $3 \mathrm{D}$ dielectric object above a $2 \mathrm{D}$ conductive rough surface. The FEM/BIM combined with KA was used in [14] to model the scattering from a $3 \mathrm{D}$ coated target above 2D PEC rough surface. The efficient and accurate modelling of inhomogeneous object buried beneath the randomly dielectric rough surface is still a challenging problem.

In this paper, a hybrid method is firstly designed for the fast and accurate solution of the 3D scattering problem related to a $3 \mathrm{D}$ inhomogeneous target buried beneath a $2 \mathrm{D}$ randomly dielectric rough surface. In order to achieve a significant reduction in CPU time and memory requirements, the asymptotic current-based KA is applied in the hybrid method to obtain the electric and magnetic current densities on the dielectric rough surface. To model the scattering from the buried inhomogeneous target, the powerful FEM/BIM is used in this paper. The multiple interactions between the target and rough surface are taken into account by updating the electric and magnetic fields on them. The accuracy of our hybrid method has been proven by comparing the results with that of the numerical method. And the effectiveness of our hybrid method is also discussed.

\section{Hybrid Theory and Formulations}

The geometry of the electromagnetic scattering problem is depicted in Figure 1. The whole space is separated into two half-spaces by the rough surface $\Gamma_{\mathrm{KA}}$, which can be defined by a function $z=f(x, y)$ with mean $\langle f(x, y)\rangle=0$. The rough surface generated by using the Monte-Carlo method based on the corresponding power spectrum function is confined to a square region $L=L_{x}=L_{y}$ on $x-y$ plane of Cartesian coordinate system. The $2 \mathrm{D}$ Gaussian roughness spectrum used in this paper is given by

$$
S\left(k_{x}, k_{y}\right)=\delta^{2} \frac{l_{x} l_{y}}{4 \pi} \exp \left(-\frac{k_{x}^{2} l_{x}^{2}+k_{y}^{2} l_{y}^{2}}{4}\right),
$$

where $\delta$ is the root-mean-square height of the rough surface, $l_{x}$ and $l_{y}$ are the correlation lengths in the $\hat{x}$ and $\hat{y}$ directions, respectively, and $k_{x}$ and $k_{y}$ are the spatial frequencies in the $\hat{x}$ and $\hat{y}$ directions, respectively.

The half-spaces above and below the rough surface are marked as region 1 and region 2 , respectively. The permittivity and permeability of region $i$ are $\varepsilon_{i}$ and $\mu_{i}$, respectively. The inhomogeneous target buried in region 2 is bounded by $\Gamma_{\mathrm{BIM}}$.

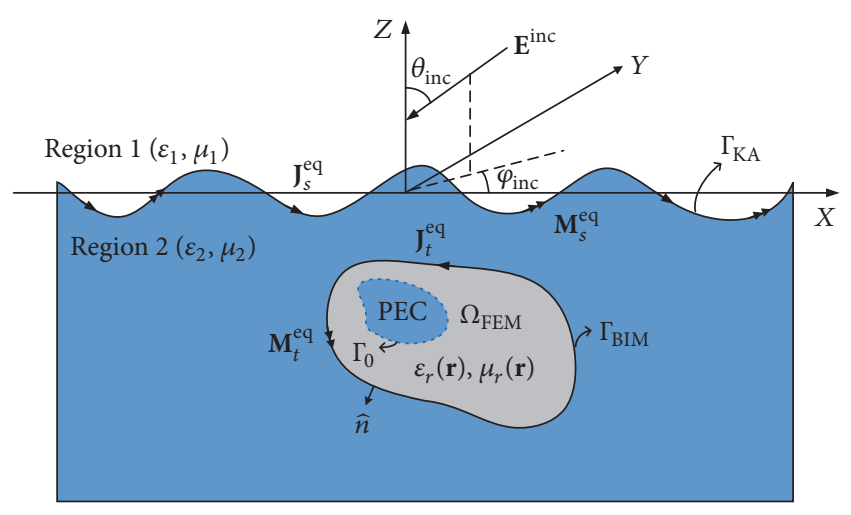

FIGURE 1: Geometry of the scattering problem of a 3D complex dielectric object buried under a $2 \mathrm{D}$ randomly dielectric rough surface.

The interior region $\Omega_{\mathrm{FEM}}$ of the target can be composed of inhomogeneous dielectric objects with complex relative permittivity $\varepsilon_{r}(\mathbf{r})$ and permeability $\mu_{r}(\mathbf{r})$, and PEC. To reduce the edge effects caused by the truncation of the finite surface length, the rough surface is illuminated by a TE polarized tapered wave [15] in this paper. The source of the tapered wave is in region 1 and the tapered wave can be expressed as

$$
\mathbf{E}^{\text {inc }}(\mathbf{r})=\exp \left[-j \mathbf{k}_{1} \cdot \mathbf{r}(1+w)\right] \exp \left(-t_{x}-t_{y}\right),
$$

where

$$
\begin{aligned}
w & =\frac{1}{k_{1}^{2}}\left[\frac{2 t_{x}-1}{g^{2} \cos ^{2} \theta_{\mathrm{inc}}}+\frac{2 t_{y}-1}{g^{2}}\right] \\
t_{x} & =\frac{\left(\cos \theta_{\mathrm{inc}} \cos \varphi_{\mathrm{inc}} x+\cos \theta_{\mathrm{inc}} \sin \varphi_{\mathrm{inc}} y+\sin \theta_{\mathrm{inc}} z\right)^{2}}{g^{2} \cos ^{2} \theta_{\mathrm{inc}}} \\
t_{y} & =\frac{\left(-\sin \varphi_{\mathrm{inc}} x+\cos \varphi_{\mathrm{inc}} y\right)^{2}}{g^{2}}
\end{aligned}
$$

where $\theta_{\text {inc }}$ and $\varphi_{\text {inc }}$ are the incident angles, $g$ is the tapered factor, and $k_{1}$ is the wavenumber in the half space above the rough surface.

Hybrid FEM/BIM is a powerful technique to deal with the inhomogeneous media problem due to the differential equation nature. As shown in Figure 1, region 2 can be decomposed into two parts by boundary $\Gamma_{\mathrm{BIM}}$, the interior region $\Omega_{\mathrm{FEM}}$, and the outer region. In FEM/BIM, the interior region $\Omega_{\mathrm{FEM}}$ is analysed by FEM and the outer region is modelled by BIM, which naturally satisfies the radiation condition. The interior field and the outer field are coupled together via the field continuity conditions on the boundary $\Gamma_{\mathrm{BIM}}$.

In the FEM region $\Omega_{\mathrm{FEM}}$, the electric field satisfies the following Helmholtz's equation:

$$
\begin{array}{r}
\nabla \times\left(\frac{1}{\mu_{r}(\mathbf{r})} \nabla \times \mathbf{E}(\mathbf{r})\right)-k_{0}^{2} \varepsilon_{r}(\mathbf{r}) \mathbf{E}(\mathbf{r})=-j k_{0} \eta_{0} \mathbf{J}(\mathbf{r}) \\
\left(\mathbf{r} \in \Omega_{\mathrm{FEM}}\right),
\end{array}
$$


where $k_{0}$ is the free-space wavenumber, $\mathbf{J}(\mathbf{r})$ is the electric current in the space, $\eta_{0}$ is the free-space intrinsic impedance, and $\mathbf{E}(\mathbf{r})$ is the total electric field.

Based on the theory of functional analysis, the equivalent variational problem can be formulated as [16]

$$
\begin{aligned}
\delta F(\mathbf{E})=0 & \\
\widehat{n} \times \mathbf{E}(\mathbf{r}) & =0 \quad \mathbf{r} \in \Gamma_{0},
\end{aligned}
$$

where

$$
\begin{aligned}
& F(\mathbf{E})=\frac{1}{2} \\
& \quad \cdot \iiint_{\Omega_{\mathrm{FEM}}}\left[\frac{1}{\mu_{r}(\mathbf{r})}(\nabla \times \mathbf{E}) \cdot(\nabla \times \mathbf{E})-k_{0}^{2} \varepsilon_{r}(\mathbf{r}) \mathbf{E} \cdot \mathbf{E}\right] d V \\
& \quad-j k_{0} \eta_{0} \oiint_{\Gamma_{\mathrm{BIM}}} \mathbf{E} \cdot\left(\widehat{n} \times \mathbf{H}_{t}^{\text {scat }}\right) d S,
\end{aligned}
$$

where $\hat{n}$ is the out-ward unit vector normal to boundary $\Gamma_{\mathrm{BIM}}$ and $\mathbf{H}_{t}^{\text {scat }}$ is the magnetic field scattered by the target on the boundary $\Gamma_{\mathrm{BIM}}$. Discretizing the FEM region with tetrahedral elements, the fields can be expanded by the vector basis function [16] $\mathbf{N}_{m}(\mathbf{r})$ :

$$
\begin{gathered}
\mathbf{E}(\mathbf{r})=\sum_{m=1}^{M} E_{m} \mathbf{N}_{m}(\mathbf{r}) \\
\mathbf{H}(\mathbf{r})=\sum_{m=1}^{M} H_{m} \mathbf{N}_{m}(\mathbf{r}),
\end{gathered}
$$

where $M$ is the number of the vector bases defined in FEM region. Then the following weak form of the matrix equation can be obtained:

$$
\begin{aligned}
& \iiint_{\Omega_{\mathrm{FEM}}}\left[\frac{1}{\mu_{\mathrm{r}}(\mathbf{r})}\left(\nabla \times \mathbf{N}_{i}\right) \cdot(\nabla \times \mathbf{E})-k_{0}^{2} \varepsilon_{r}(\mathbf{r}) \mathbf{N}_{i} \cdot \mathbf{E}\right] d V \\
& -j k_{0} \eta_{0} \iint_{\Gamma_{\mathrm{BIM}}} \mathbf{N}_{i} \cdot\left(\widehat{n} \times \mathbf{H}_{t}^{\text {scat }}\right) d S=0 .
\end{aligned}
$$

The outer region can be modelled by the integral equation method. The total electric field $\mathbf{E}(\mathbf{r})$ in the outer region satisfies the following integral equation:

$$
\mathbf{E}(\mathbf{r})-\mathbf{E}_{t}^{\mathrm{scat}}(\mathbf{r})=\mathbf{E}_{s}^{\mathrm{scat}}(\mathbf{r}),
$$

where $\mathbf{E}_{s}^{\text {scat }}(\mathbf{r})$ is the field excited by the equivalent surface electric and magnetic currents $\left(\mathrm{J}_{s}^{\mathrm{eq}}\right.$ and $\left.\mathbf{M}_{s}^{\mathrm{eq}}\right)$ on the rough surface, which act as the source illuminating on the target. $\mathbf{E}_{t}^{\text {scat }}(\mathbf{r})$ is the field excited by the equivalent surface electric and magnetic currents $\left(\mathbf{J}_{t}^{\mathrm{eq}}\right.$ and $\mathbf{M}_{t}^{\mathrm{eq}}$ ) on the boundary $\Gamma_{\mathrm{BIM}}$. The fields $\mathbf{E}_{t}^{\text {scat }}(\mathbf{r})$ and $\mathbf{E}_{s}^{\text {scat }}(\mathbf{r})$ can be obtained from the following expressions, respectively:

$$
\begin{aligned}
& \mathbf{E}_{t}^{\text {scat }}(\mathbf{r})=\eta_{2} \mathbf{L}_{2}\left(\mathbf{J}_{t}^{\mathrm{eq}}\right)-\mathbf{K}_{2}\left(\mathbf{M}_{t}^{\mathrm{eq}}\right) \\
& \mathbf{E}_{s}^{\text {scat }}(\mathbf{r})=-\eta_{2} \mathbf{L}_{2}\left(\mathbf{J}_{s}^{\mathrm{eq}}\right)+\mathbf{K}_{2}\left(\mathbf{M}_{s}^{\mathrm{eq}}\right) .
\end{aligned}
$$

The operators $\mathbf{L}_{i}$ and $\mathbf{K}_{i}$ are defined as

$$
\begin{gathered}
\mathbf{L}_{i}(\mathbf{X})=-j k_{i} \iint_{\Gamma} \mathbf{X}\left(\mathbf{r}^{\prime}\right) G_{i}\left(\mathbf{r}, \mathbf{r}^{\prime}\right)+\frac{1}{k_{i}^{2}} \nabla^{\prime} \\
\cdot \mathbf{X}\left(\mathbf{r}^{\prime}\right) \nabla G_{i}\left(\mathbf{r}, \mathbf{r}^{\prime}\right) d \Gamma^{\prime} \\
\mathbf{K}_{i}(\mathbf{X})=-\iint_{\Gamma} \mathbf{X}\left(\mathbf{r}^{\prime}\right) \times \nabla G_{i}\left(\mathbf{r}, \mathbf{r}^{\prime}\right) d \Gamma^{\prime}
\end{gathered}
$$

The symbols $\eta_{i}, k_{i}$, and $G_{i}$ are the complex impedance, the wavenumber, and Green's function associated with region $i$, respectively. The expression of the Green's function $G_{i}$ is given by

$$
G_{i}\left(\mathbf{r}, \mathbf{r}^{\prime}\right)=\frac{e^{-j k_{i}\left|\mathbf{r}-\mathbf{r}^{\prime}\right|}}{4 \pi\left|\mathbf{r}-\mathbf{r}^{\prime}\right|},
$$

where $\mathbf{r}^{\prime}$ and $\mathbf{r}$ are the position vectors of the source and observation points, respectively.

On the boundary $\Gamma_{\mathrm{BIM}}$, the equivalent electric and magnetic currents $\left(\mathbf{J}_{t}^{\mathrm{eq}}\right.$ and $\left.\mathbf{M}_{t}^{\mathrm{eq}}\right)$ have the relation with surface fields as $\mathbf{J}_{t}^{\mathrm{eq}}=\widehat{n} \times \mathbf{H}_{t}^{\text {scat }}$ and $\mathbf{M}_{t}^{\mathrm{eq}}=-\widehat{n} \times \mathbf{E}_{t}^{\text {scat }}$, respectively. Then the electric and magnetic currents on the boundary can be discretized as

$$
\begin{aligned}
\mathbf{J}_{t}^{\mathrm{eq}}(\mathbf{r}) & =\sum_{n=1}^{N_{t}} H_{n} \mathbf{f}_{n}(\mathbf{r}) \\
\mathbf{M}_{t}^{\mathrm{eq}}(\mathbf{r}) & =\sum_{n=1}^{N_{t}} E_{n} \mathbf{f}_{n}(\mathbf{r}),
\end{aligned}
$$

where $\mathbf{f}_{n}(\mathbf{r})$ is the Rao-Wilton-Glisson (RWG) basis functions which have relation with $\mathbf{N}_{n}(\mathbf{r})$ as $\mathbf{f}_{n}(\mathbf{r})=-\widehat{n} \times \mathbf{N}_{n}(\mathbf{r})$. When MoM is applied, the following matrix equations can be obtained by discretizing (9):

$$
[P]\{E\}+[Q]\{H\}=\{b\},
$$

where $[P]$ and $[Q]$ are the impedance matrices and $\{b\}$ is the vector related to the fields scattered from the rough surface. And they can be obtained by the following expressions:

$$
\begin{aligned}
& P_{m n} \\
& =\left\langle\mathbf{f}_{m}(\mathbf{r}), \mathbf{N}_{n}(\mathbf{r})+\int_{S_{n}} \mathbf{f}_{n}\left(\mathbf{r}^{\prime}\right) \times \nabla^{\prime} G_{2}\left(\mathbf{r}, \mathbf{r}^{\prime}\right) d S^{\prime}\right\rangle \\
& Q_{m n} \\
& =\left\langle\mathbf{f}_{m}(\mathbf{r}), j k_{2} \eta_{2} \int_{S_{n}} \mathbf{f}_{n}\left(\mathbf{r}^{\prime}\right) G_{2}\left(\mathbf{r}, \mathbf{r}^{\prime}\right) d S^{\prime}\right\rangle \\
& \quad+\left\langle\mathbf{f}_{m}(\mathbf{r}), \frac{j \eta_{2}}{k_{2}} \int_{S_{n}}\left[\nabla^{\prime} \cdot \mathbf{f}_{n}\left(\mathbf{r}^{\prime}\right)\right] \nabla G_{2}\left(\mathbf{r}, \mathbf{r}^{\prime}\right) d S^{\prime}\right\rangle \\
& b_{m}=\left\langle\mathbf{f}_{m}(\mathbf{r}), \mathbf{E}_{s}^{\text {scat }}\left(\mathbf{r}^{\prime}\right)\right\rangle .
\end{aligned}
$$


Then we can obtain the final matrix equation for the scattering problem of the inhomogeneous target buried under the dielectric rough surface as

$$
\left[\begin{array}{ccc}
K_{\mathrm{II}} & K_{\mathrm{IS}} & 0 \\
K_{\mathrm{SI}} & K_{\mathrm{SS}} & C \\
0 & P & Q
\end{array}\right]\left\{\begin{array}{l}
E_{\mathrm{I}} \\
E_{\mathrm{S}} \\
H_{\mathrm{S}}
\end{array}\right\}=\left\{\begin{array}{l}
0 \\
0 \\
b
\end{array}\right\},
$$

where $\left[K_{\mathrm{II}}\right],\left[K_{\mathrm{IS}}\right],\left[K_{\mathrm{SI}}\right]$, and $\left[K_{\mathrm{SS}}\right]$ are the FEM matrices. The subscript $\mathrm{I}$ is denoting in the interior region $\Omega_{\mathrm{FEM}}$ and subscript $S$ is denoting on the boundary $\Gamma_{\mathrm{BIM}}$. $[C]$ is the coupling matrix between FEM and BIM. $[K]$ and $[C]$ can be represented by the following expressions, respectively:

$$
\begin{aligned}
K_{m n}= & \iiint_{\Omega} \frac{1}{\mu_{r}}\left(\nabla \times \mathbf{N}_{m}\right) \cdot\left(\nabla \times \mathbf{N}_{n}\right)-k_{0}^{2} \varepsilon_{r} \mathbf{N}_{m} \\
& \cdot \mathbf{N}_{n} d V \\
C_{m n}= & j k_{0} \eta_{0} \iint_{\Gamma_{m}} \mathbf{N}_{m} \cdot\left(\widehat{n} \times \mathbf{N}_{n}\right) d S .
\end{aligned}
$$

Note that the effects of the rough surface are considered in (17) as terms $[b]$. To get the field $\mathbf{E}_{s}^{\text {scat }}$ scattered from the rough surface, we should firstly compute the equivalent surface electric and magnetic currents $\left(\mathbf{J}_{s}^{\mathrm{eq}}\right.$ and $\left.\mathbf{M}_{s}^{\mathrm{eq}}\right)$ on the rough surface. They can be rigorously achieved by solving the following integral equations with MoM:

$$
\begin{aligned}
& -\eta_{2} \mathbf{L}_{2}\left(\mathbf{J}_{s}^{\mathrm{eq}}\right)-\eta_{1} \mathbf{L}_{1}\left(\mathbf{J}_{s}^{\mathrm{eq}}\right)+\mathbf{K}_{2}\left(\mathbf{M}_{s}^{\mathrm{eq}}\right)+\mathbf{K}_{1}\left(\mathbf{M}_{s}^{\mathrm{eq}}\right) \\
& =\mathbf{E}^{\mathrm{inc}}-\mathbf{E}_{t}^{\mathrm{scat}} \\
& -\mathbf{K}_{2}\left(\mathbf{J}_{s}^{\mathrm{eq}}\right)-\mathbf{K}_{1}\left(\mathrm{~J}_{s}^{\mathrm{eq}}\right)-\frac{1}{\eta_{1}} \mathbf{L}_{1}\left(\mathbf{M}_{s}^{\mathrm{eq}}\right)-\frac{1}{\eta_{2}} \mathbf{L}_{2}\left(\mathbf{M}_{s}^{\mathrm{eq}}\right) \\
& =\mathbf{H}^{\mathrm{inc}}-\mathbf{H}_{t}^{\mathrm{scat}} .
\end{aligned}
$$

However, solving these integral equations with MoM is very time-consuming and requires a great amount of storage memory, especially for 3D electrically large rough surface. To reduce the computation time and memory requirement, analytical KA is a good choice.

Based on the superposition principle, the electric and magnetic currents on the rough surface can be represented by the combination of two independent parts as

$$
\begin{aligned}
\mathbf{J}_{s}^{\mathrm{eq}} & =\mathbf{J}_{s}^{\mathrm{inc}}+\mathbf{J}_{s}^{\mathrm{obj}} \\
\mathbf{M}_{s}^{\mathrm{eq}} & =\mathbf{M}_{s}^{\mathrm{inc}}+\mathbf{M}_{s}^{\mathrm{obj}},
\end{aligned}
$$

where $\mathbf{J}_{s}^{\text {inc }}$ and $\mathbf{M}_{s}^{\mathrm{inc}}$ are the electric and magnetic currents induced by the incident wave in the absence of the buried target. $\mathbf{J}_{s}^{\mathrm{obj}}$ and $\mathbf{M}_{s}^{\mathrm{obj}}$ are the electric and magnetic currents generated by the fields scattered by the buried target in the absence of the incident wave source.
According to the KA theory and equivalent principle, the electric and magnetic currents $\left(\mathbf{J}_{s}^{\text {inc }}\right.$ and $\left.\mathbf{M}_{s}^{\text {inc }}\right)$ at any point $\mathbf{r}$ can be expressed as

$$
\begin{aligned}
\mathbf{J}_{s}^{\mathrm{inc}} & =\widehat{n}_{s} \times\left[\frac{\left(1+R_{U}^{\mathrm{TM}}\right)\left(\widehat{p}_{i} \cdot \mathbf{E}^{\mathrm{inc}}\right)}{\eta_{1}} \widehat{q}_{i}\right. \\
& \left.-\frac{\left(\widehat{q}_{i} \cdot \mathbf{E}^{\mathrm{inc}}\right)}{\eta_{1}}\left(\widehat{p}_{i}-R_{U}^{\mathrm{TE}} \widehat{k}_{r} \times \widehat{q}_{i}\right)\right], \\
\mathbf{M}_{s}^{\mathrm{inc}} & =-\widehat{n}_{s} \times\left[\left(1+R_{U}^{\mathrm{TE}}\right)\left(\widehat{q}_{i} \cdot \mathbf{E}^{\mathrm{inc}}\right) \widehat{q}_{i}\right. \\
& \left.+\left(\widehat{p}_{i} \cdot \mathbf{E}^{\mathrm{inc}}\right)\left(\widehat{p}_{i}-R_{U}^{\mathrm{TM}} \widehat{k}_{r} \times \widehat{q}_{i}\right)\right],
\end{aligned}
$$

where $\left(\widehat{p}_{i}, \widehat{q}_{i}, \widehat{k}_{\text {inc }}\right)$ is a local orthonormal basis established at any point $\mathbf{r}$ on the rough surface. The symbol $\widehat{k}_{\text {inc }}$ is the unit incident wave vector. The unit vectors $\widehat{p}_{i}$ and $\widehat{q}_{i}$ are the local perpendicular and parallel polarization vectors, respectively, which can be defined as

$$
\begin{aligned}
& \widehat{q}_{i}(\mathbf{r})=\frac{\widehat{k}_{\mathrm{inc}} \times \widehat{n}_{s}(\mathbf{r})}{\left\|\widehat{k}_{\mathrm{inc}} \times \widehat{n}_{s}(\mathbf{r})\right\|} \\
& \widehat{p}_{i}(\mathbf{r})=\widehat{q}_{i}(\mathbf{r}) \times \widehat{k}_{\mathrm{inc}} .
\end{aligned}
$$

The symbol $\widehat{k}_{r}$ is the unit local reflection direction vector. Based on the tangent plane approximation, $\widehat{k}_{r}=\widehat{k}_{\text {inc }}-2 \widehat{n}_{s}\left(\widehat{n}_{s}\right.$. $\left.\widehat{k}_{\text {inc }}\right) \cdot R_{U}^{\mathrm{TE}}$ and $R_{U}^{\mathrm{TM}}$ are the local Fresnel reflection coefficients of TE polarization and TM polarization, respectively, which can be expressed as

$$
\begin{gathered}
R_{U}^{\mathrm{TE}}=\frac{\cos \theta_{\mathrm{li}}-\sqrt{\varepsilon_{2 r}-\sin ^{2} \theta_{\mathrm{li}}}}{\cos \theta_{\mathrm{li}}+\sqrt{\varepsilon_{2 r}-\sin ^{2} \theta_{\mathrm{li}}}} \\
R_{U}^{\mathrm{TM}}=\frac{\varepsilon_{2 r} \cos \theta_{\mathrm{li}}-\sqrt{\varepsilon_{2 r}-\sin ^{2} \theta_{\mathrm{li}}}}{\varepsilon_{2 r} \cos \theta_{\mathrm{li}}+\sqrt{\varepsilon_{2 r}-\sin ^{2} \theta_{\mathrm{li}}}},
\end{gathered}
$$

where $\theta_{\text {li }}$ is the local incident angle on the rough surface which satisfies $\cos \theta_{\mathrm{li}}=-\widehat{n}_{s} \cdot \widehat{k}_{i}$ and $\varepsilon_{2 r}$ is the relative permittivity of region 2 .

Similarly, the electric and magnetic currents $\left(J_{s}^{\text {obj }}\right.$ and $\mathbf{M}_{s}^{\mathrm{obj}}$ ) generated by the fields scattered by the buried target can be expressed as

$$
\begin{gathered}
\mathbf{J}_{s}^{\mathrm{obj}}=\widehat{n}_{s} \times\left[\frac{\left(1+R_{D}^{\mathrm{TM}}\right)\left(\widehat{p}_{s} \cdot \mathbf{E}_{t}^{\mathrm{scat}}\right)}{\eta_{2}} \widehat{q}_{s}\right. \\
\left.-\frac{\left(\widehat{q}_{s} \cdot \mathbf{E}_{t}^{\mathrm{scat}}\right)}{\eta_{2}}\left(\widehat{p}_{s}-R_{D}^{\mathrm{TE}} \widehat{k}_{r s} \times \widehat{q}_{s}\right)\right], \\
\mathbf{M}_{s}^{\mathrm{obj}}=-\widehat{n}_{s} \times\left[\left(1+R_{D}^{\mathrm{TE}}\right)\left(\widehat{q}_{s} \cdot \mathbf{E}_{t}^{\mathrm{scat}}\right) \widehat{q}_{s}\right. \\
\left.+\left(\widehat{p}_{s} \cdot \mathbf{E}_{t}^{\mathrm{scat}}\right)\left(\widehat{p}_{s}-R_{D}^{\mathrm{TM}} \widehat{k}_{r s} \times \widehat{q}_{s}\right)\right],
\end{gathered}
$$




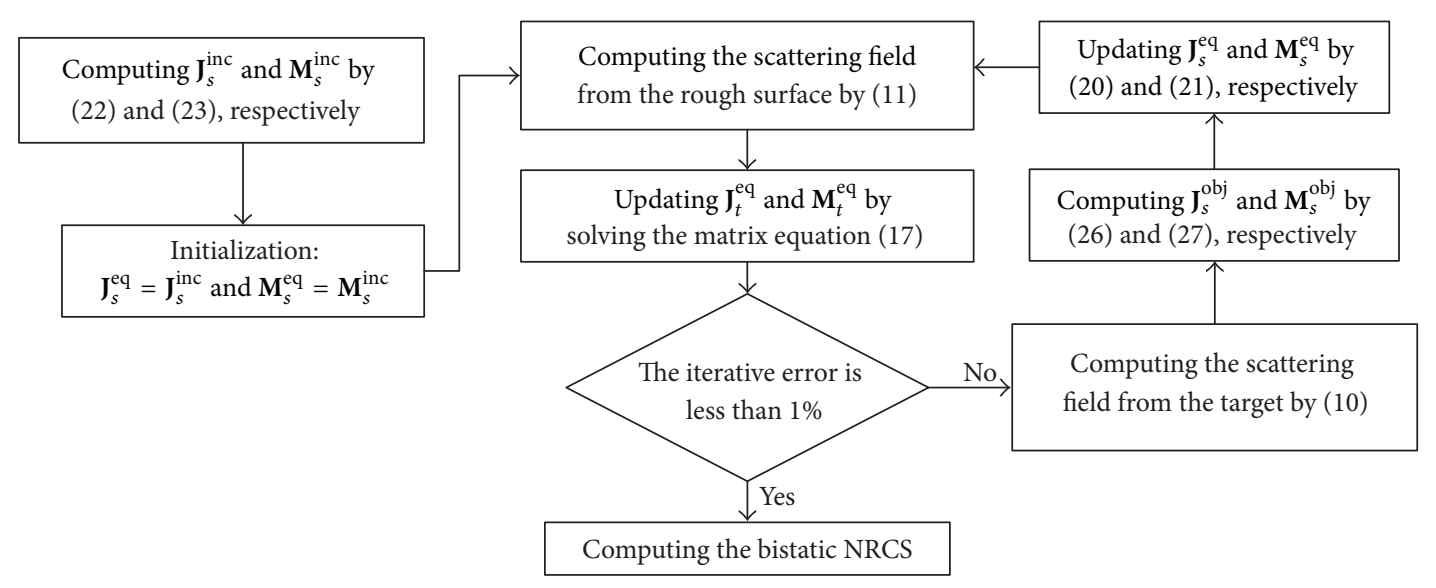

FIGURE 2: The flowchart of the iterative method.

where $\left(\widehat{p}_{s}, \widehat{q}_{s}, \widehat{r}_{s}\right)$ is a local orthonormal basis established at any point $\mathbf{r}$ on the rough surface. $\widehat{r}_{s}$ is the unit vector from the source point $\mathbf{r}^{\prime}$ on the target to the field point $\mathbf{r}$ on the rough surface, which satisfies $\widehat{r}_{s}=\left(\mathbf{r}-\mathbf{r}^{\prime}\right) /\left\|\mathbf{r}-\mathbf{r}^{\prime}\right\|$. The unit vectors $\widehat{p}_{s}$ and $\widehat{q}_{s}$ are the local perpendicular and parallel polarization vectors, respectively, which can be defined as

$$
\begin{aligned}
& \widehat{q}_{s}(\mathbf{r})=\frac{\widehat{r}_{s} \times \widehat{n}_{s}(\mathbf{r})}{\left\|\widehat{r}_{s} \times \widehat{n}_{s}(\mathbf{r})\right\|} \\
& \widehat{p}_{s}(\mathbf{r})=\widehat{q}_{s}(\mathbf{r}) \times \widehat{r}_{s},
\end{aligned}
$$

where $\widehat{k}_{\mathrm{rs}}$ is the unit local reflection direction of the wave induced by the electric and magnetic currents on the target, which act as another incident source illuminating on the rough surface from the bottom. $R_{D}^{\mathrm{TE}}$ and $R_{D}^{\mathrm{TM}}$ are the local Fresnel reflection coefficients of TE polarization and TM polarization, respectively, which can be expressed as

$$
\begin{aligned}
& R_{D}^{\mathrm{TE}}=\frac{\sqrt{\varepsilon_{2 r}} \cos \theta_{\mathrm{ls}}-\sqrt{1-\varepsilon_{2 r} \sin ^{2} \theta_{\mathrm{ls}}}}{\sqrt{\varepsilon_{2 r}} \cos \theta_{\mathrm{ls}}+\sqrt{1-\varepsilon_{2 r} \sin ^{2} \theta_{\mathrm{ls}}}} \\
& R_{D}^{\mathrm{TM}}=\frac{\cos \theta_{\mathrm{ls}}-\sqrt{\varepsilon_{2 r}} \sqrt{1-\varepsilon_{2 r} \sin ^{2} \theta_{\mathrm{ls}}}}{\cos \theta_{\mathrm{ls}}+\sqrt{\varepsilon_{2 r}} \sqrt{1-\varepsilon_{2 r} \sin ^{2} \theta_{\mathrm{ls}}}},
\end{aligned}
$$

where $\theta_{\mathrm{ls}}$ is the local incident angle and satisfies $\cos \theta_{\mathrm{ls}}=$ $\widehat{n}_{s}(\mathbf{r}) \cdot \widehat{r}_{s}$.

Then the scattering information from the rough surface can be obtained by (20), (22), and (26), if the scattering field $\mathbf{E}_{t}^{\text {scat }}$ from the target is calculated beforehand. It should be pointed out that, since the scattering from the rough surface is considered by KA, the limitation of KA [17] should be taken into account in our hybrid method; namely, the curvature radius $\rho$ of the rough surface should be very large compared to the wavelength of the incident wave, $\rho \gg \lambda$.

The multiple interaction between the rough surface and the buried target can be taken into account by an iterative approach. To give a clearer depiction, the iterative process in our method is shown in Figure 2. The iterative error at the $i$ th iteration is defined as

$$
\gamma_{i}=\frac{\left\|\mathbf{J}_{t}^{i}-\mathbf{J}_{t}^{i-1}\right\|}{\left\|\mathbf{J}_{t}^{i}\right\|}
$$

Once the scattering information from the rough surface is obtained, the bistatic normalized radar cross-section (NRCS) can be calculated by

$$
\mathrm{NRCS}=\lim _{r \rightarrow \infty} r^{2} \frac{\left\|\mathbf{E}^{\text {scat }}\right\|^{2}}{P_{\text {inc }}},
$$

where $\mathbf{E}^{\text {scat }}$ is the field scattered by the rough surface at any point $\mathbf{r}$ in the region 1 , which can be obtained with the following expression:

$$
\mathbf{E}^{\text {scat }}=\eta_{1} \mathbf{L}_{1}\left(\mathbf{J}_{s}^{\mathrm{eq}}\right)-\mathbf{K}_{1}\left(\mathbf{M}_{s}^{\mathrm{eq}}\right) .
$$

The symbol $P^{\text {inc }}$ is the incident beam power which can be given by

$$
P^{\text {inc }}=\frac{\pi}{2} g^{2} \cos \theta_{\text {inc }}\left[1-\frac{1+\cos ^{2} \theta_{\text {inc }}+2 \tan ^{2} \theta_{\text {inc }}}{2 k_{1}^{2} g^{2} \cos ^{2} \theta_{\text {inc }}}\right] .
$$

\section{Numerical Results}

In this part, several numerical simulations are considered to demonstrate the algorithm's ability to deal with the scattering from the inhomogeneous target buried beneath a dielectric rough surface. The effectiveness of our proposed method is also illustrated. In all examples, the region 1 is considered as free space. In addition, all sizes are given in terms of freespace wavelength $\lambda$ which is equal to $1 \mathrm{~m}$ in this paper unless otherwise specified. Finally, the relative permittivity of region 2 is $\varepsilon_{2 r}=(2.0,-0.01)$.

Our numerical code is firstly applied to calculate the bistatic NRCS of a 3D PEC sphere buried under a Gaussian dielectric rough surface. The radius of the sphere is $0.5 \mathrm{~m}$ and the burial depth is $4.0 \mathrm{~m}$. The incident angles are $\theta_{\text {inc }}=0^{\circ}$ and $\varphi_{\text {inc }}=0^{\circ}$. The length of the truncated rough surface is $L=$ 


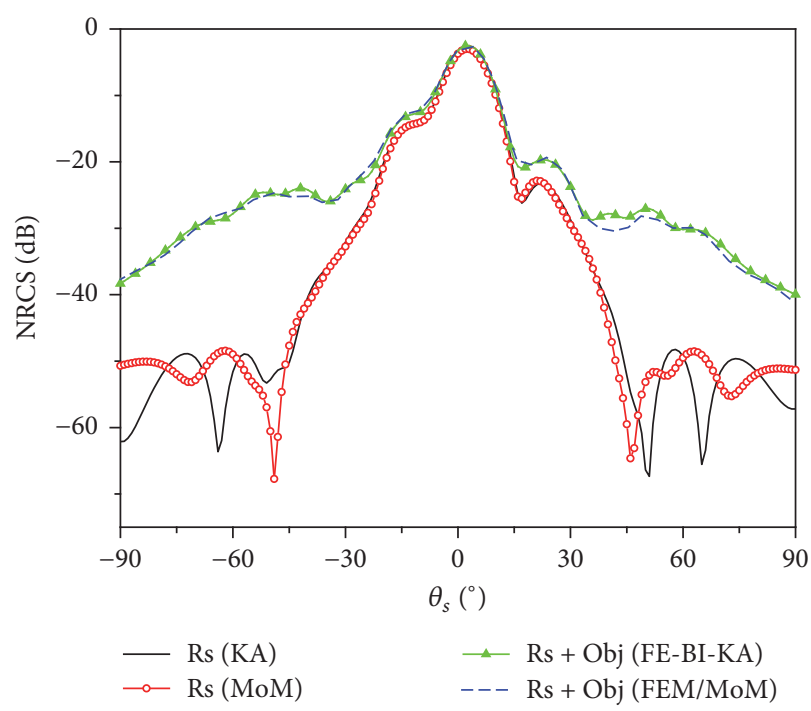

(a)

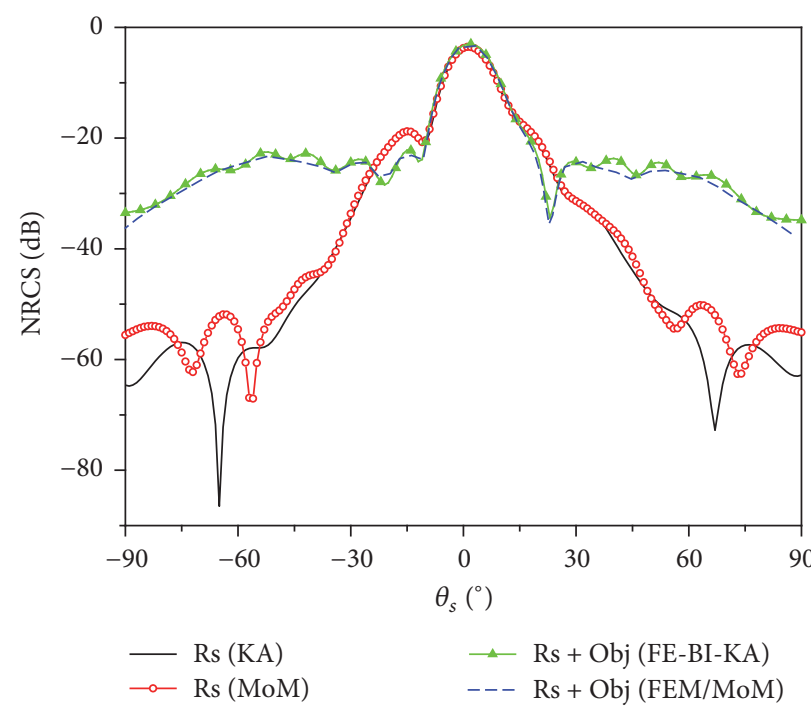

(b)

FIgURE 3: Bistatic NRCS of a 3D PEC sphere buried under dielectric rough surface: (a) $X-Z$ Plane and (b) $Y-Z$ Plane.

TABLE 1: Comparisons of the computing resource for different methods.

\begin{tabular}{lccc}
\hline Method & $\begin{array}{c}\text { Number of } \\
\text { unknowns }\end{array}$ & $\begin{array}{c}\text { Memory } \\
\text { requirement }(\mathrm{G})\end{array}$ & $\begin{array}{c}\text { Solving } \\
\text { time }(\mathrm{s})\end{array}$ \\
\hline FEM/MoM & 83836 & 107.34 & 3807 \\
New method & 12186 & 1.43 & 127 \\
\hline
\end{tabular}

Calculated by a computer with a $2.8 \mathrm{GHz}$ processor (AMD Opteron (tm) Processor 4280), RAM 128 GB.

$9.0 \mathrm{~m}$. The tapering parameter of the tapered incident wave is $g=L / 4$. The root-mean-square height and the correlation length of the rough surface are $\delta=2.0 \mathrm{~m}$ and $l_{x}=l_{y}=l=$ $0.1 \mathrm{~m}$, respectively.

Figures 3(a) and 3(b) show the bistatic NRCS as a function of scattering angle $\theta_{s}$ in $X-Z$ and $Y-Z$ planes, respectively. And the results are obtained with single rough surface sample. In the legends, "Rs + Object" denotes the model of an object buried under the rough surface. "Rs" denotes the model of the rough surface only. Figure 3 shows that the results obtained by our method agree with solutions of FEM/MoM very well. It can also be observed that the total NRCS is mainly contributed by the rough surface within the scattering angles $\theta_{s} \in\left(-15^{\circ}, 15^{\circ}\right)$, while in other scattering angles, the total NRCS is mainly contributed by the buried target.

Table 1 shows the comparisons of the computing resource for a single rough surface sample for different methods. The matrix of FEM/BIM is stored with compress sparse row format, and the parallel LU decomposition is used to solve the matrix equation. Table 1 indicates that the number of unknowns in our method is reduced to $14.5 \%$ of that in FEM/MoM, and the memory requirement and solving time are reduced to $1.3 \%$ and $3.3 \%$, respectively. The result shows that our method is exact and efficient in dealing with the scattering from the target buried beneath rough surface.

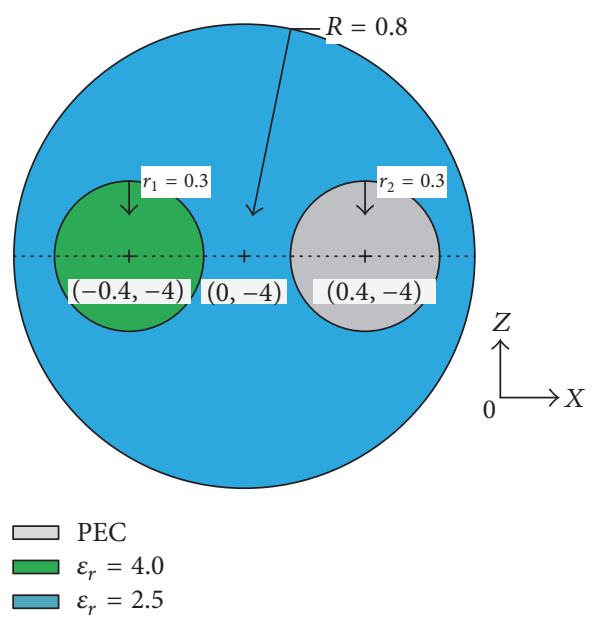

FIGURE 4: Cross-section of an inhomogeneous sphere.

To further demonstrate the ability of our method to deal with the composite scattering from the inhomogeneous target buried under rough surface, the scattering from a 3D inhomogeneous sphere buried under a dielectric rough surface, as shown in Figure 4, is calculated. The default parameters are set as follows: the incident angles are $\theta_{\text {inc }}=15^{\circ}$ and $\varphi_{\text {inc }}=0^{\circ}$, the truncated length of the rough surface is $L=$ $10.5 \mathrm{~m}$, and the root-mean-square height and the correlation length of the rough surface are $\delta=1.5 \mathrm{~m}$ and $l_{x}=l_{y}=l=$ $0.1 \mathrm{~m}$, respectively.

Figures 5(a) and 5(b) show the bistatic NRCS as a function of scattering angle $\theta_{s}$ in $X-Z$ and $Y-Z$ planes, respectively. And the results are obtained with single rough surface sample. It can be seen from Figure 5 that the results obtained by our hybrid method agree with solutions of FEM/MoM very 


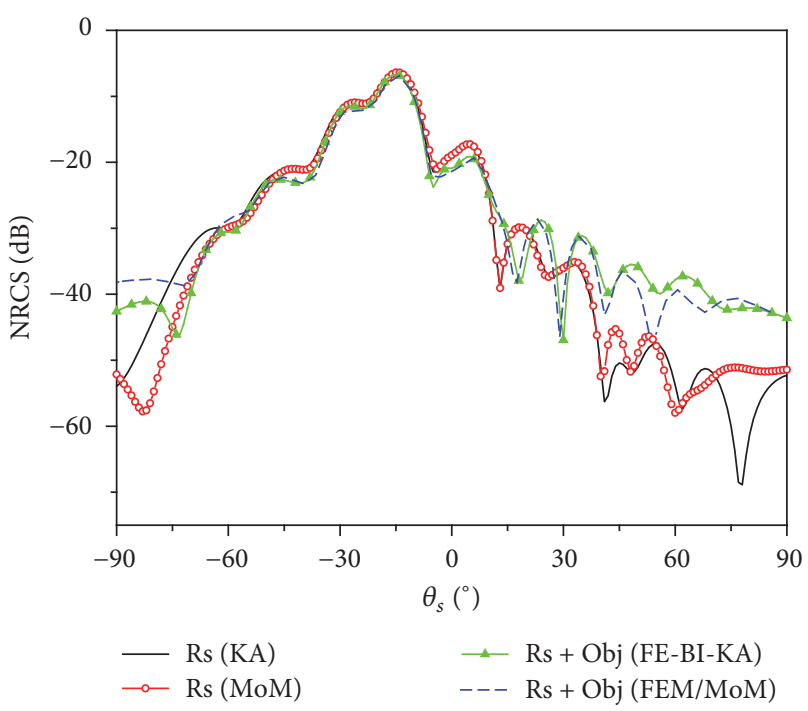

(a)

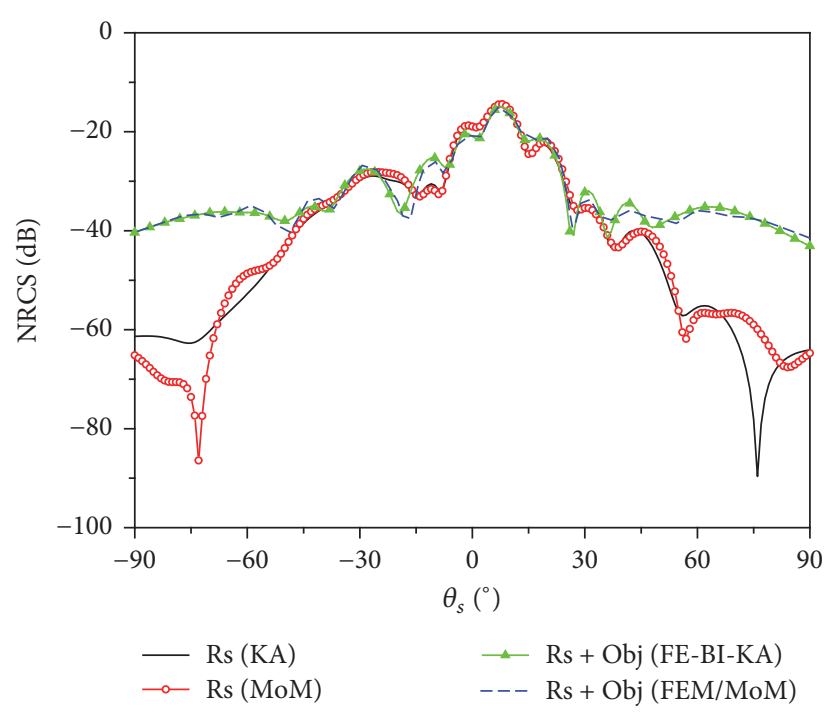

(b)

FIGURE 5: Bistatic NRCS of a 3D inhomogeneous sphere buried under dielectric rough surface: (a) $X-Z$ Plane and (b) $Y-Z$ Plane.

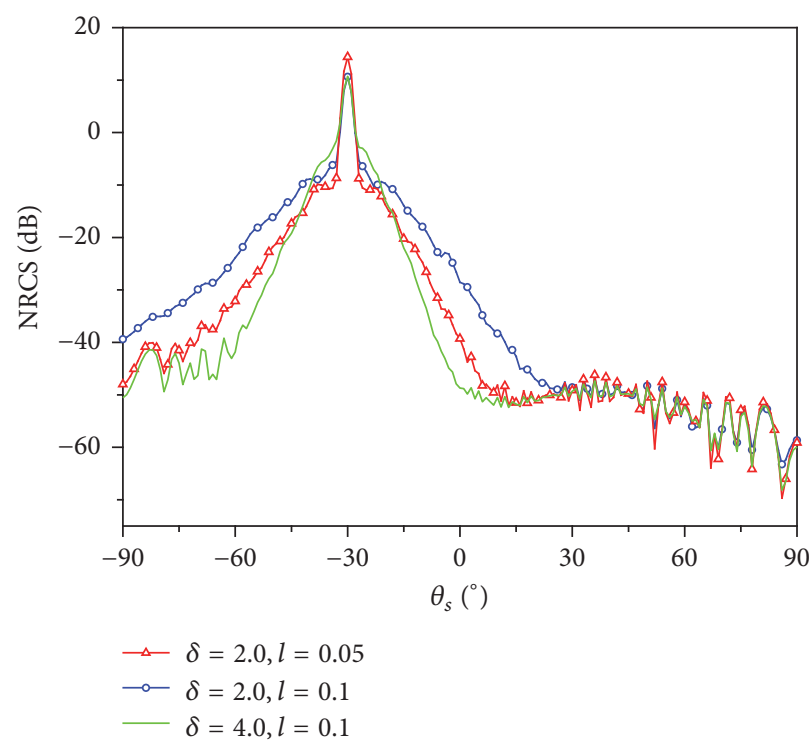

(a)

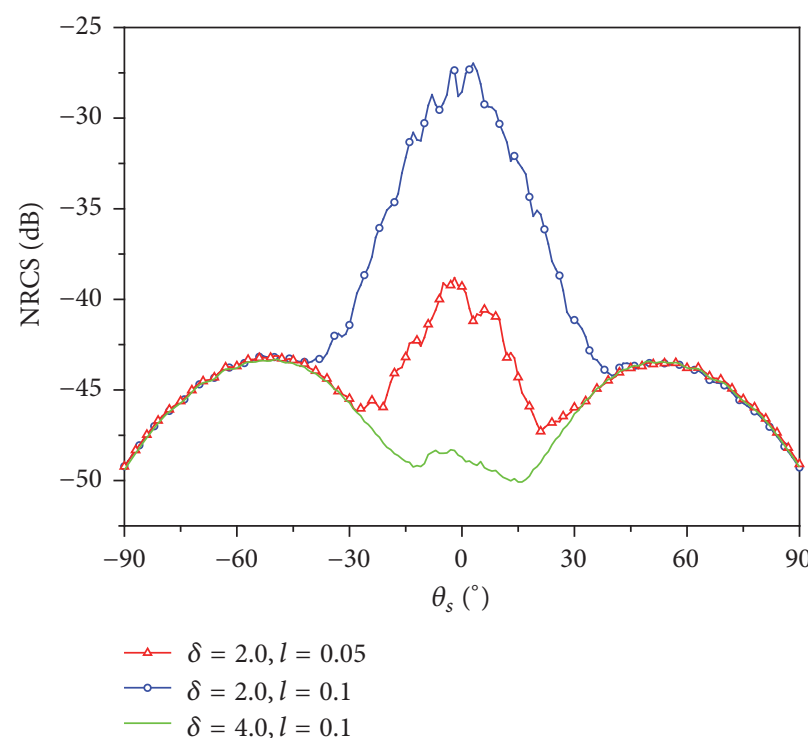

(b)

FIGURE 6: Scattering results of a 3D inhomogeneous sphere buried under dielectric rough surface with different $\delta$ and $l$ : (a) $X-Z$ Plane and (b) $Y$-Z Plane.

well, demonstrating the ability of our method to deal with the scattering from inhomogeneous target.

In the third example, the influence of the surface roughness on the scattering properties of the inhomogeneous sphere buried under dielectric rough surface is studied. The parameters of the inhomogeneous sphere are shown in Figure 4. The other parameters are set as follows: the length of the rough surface is $L=50 \mathrm{~m}$, the incident angles are $\theta_{\text {inc }}=30^{\circ}$ and $\varphi_{\text {inc }}=0^{\circ}$. The parameters of the root-meansquare height $\delta$ and the correlation length $l_{x}=l_{y}=l$ of the rough surface can be found in the Figure 6.
The curves in Figure 6 present the influence of the surface roughness on the scattering properties of the inhomogeneous sphere buried under dielectric rough surface. The results are obtained by taking the average of 50 rough surface realizations. It can be seen from Figure 6(a) that the scattering coefficients have a small change with the change of the roughness in the scattering directions defined by $\theta_{s} \in$ $\left[30^{\circ}, 90^{\circ}\right]$. Within the scattering angles $\theta_{s} \in\left[-90^{\circ}, 30^{\circ}\right]$, the scattering coefficient decreases in the specular direction with the increase in the root-mean-square height of the rough surface but increases in other scattering directions in the $X-Z$ 
Plane. In the $Y-Z$ Plane, the NRCS increase evidently with the increase in the root-mean-square height of the rough surface in the scattering directions defined by $\theta_{s} \in\left[-45^{\circ}, 45^{\circ}\right]$. The increase in the correlation length weakens the NRCS in the scattering directions $\theta_{s} \in\left[-30^{\circ}, 30^{\circ}\right]$ in the $Y-Z$ Plane.

\section{Conclusions}

In this paper, an efficient hybrid method has been presented to study the scattering from a $3 \mathrm{D}$ inhomogeneous object buried beneath a $2 \mathrm{D}$ dielectric rough surface. The electric and magnetic currents on the rough surface are analytically obtained by $\mathrm{KA}$, which can greatly reduce the computing resource. The hybrid vector FEM/BIM, a powerful technique to model complex dielectric problem, is chosen to calculate the electric and magnetic currents on the inhomogeneous object. The matrix of FEM/BIM is stored with compress sparse row format, and the parallel LU decomposition is used to solve the matrix. The validity of our hybrid method is verified by numerical method. The effectiveness of our method is also illustrated. The results show that the memory requirement in our hybrid method is greatly reduced compared with the numerical method, and the computational time is also greatly reduced. The ability of our method to deal with the scattering inhomogeneous object buried beneath rough surface is also demonstrated.

\section{Conflicts of Interest}

The authors declare that they have no conflicts of interest.

\section{Acknowledgments}

This work was supported by the National Natural Science Foundation for Distinguished Young Scholars of China (Grant no. 61225002) and the Foundation for Innovative Research Groups of the National Natural Science Foundation of China (Grant no. 61621005).

\section{References}

[1] W. Xande, G. Yow-Beng, and L. Le-Wei, "TE scattering from PEC object partially embedded at dielectric rough surface interface," in Proceedings of The Fifth International Kharkov Symposium on Physics and Engineering of Microwaves, Millimeter, and Submillimeter Waves (IEEE Cat. No.04EX828), vol. 1, pp. 287-289, Kharkov, Ukraine, 2004.

[2] A.-Q. Wang, L.-X. Guo, and C. Chai, "Application of a multiregion model to the em scattering from a rough surface with or without a target above it," Chinese Physics B, vol. 20, no. 5, Article ID 050201, 2011.

[3] Y. Altuncu, "A numerical method for electromagnetic scattering by 3-D dielectric objects buried under 2-D Locally rough surfaces," Institute of Electrical and Electronics Engineers. Transactions on Antennas and Propagation, vol. 63, no. 8, pp. 36343643, 2015.

[4] X.-C. Ren and L.-X. Guo, "Investigation on electromagnetic scattering from rough soil surface of layered medium using the small perturbation method," Chinese Physics B, vol. 17, no. 7, pp. 2491-2498, 2008.

[5] M. A. Fiaz, F. Frezza, C. Ponti, and G. Schettini, "Electromagnetic scattering by a circular cylinder buried below a slightly rough Gaussian surface," Journal of the Optical Society of America A: Optics and Image Science, and Vision, vol. 31, no. 1, pp. 26-34, 2014.

[6] D. E. Lawrence and K. Sarabandi, "Electromagnetic scattering from a dielectric cylinder buried beneath a slightly rough surface," Institute of Electrical and Electronics Engineers. Transactions on Antennas and Propagation, vol. 50, no. 10, pp. 13681376, 2002.

[7] Y.-M. Zhang, Y.-H. Wang, and L.-X. Guo, "Study of scattering from time-varying Gerstners sea surface using second-order small slope approximation," Chinese Physics B, vol. 19, no. 5, pp. 0541031-0541039, 2010.

[8] C. Bourlier, N. Pinel, and G. Kubické, "Propagation-insidelayer-expansion method combined with physical optics for scattering by coated cylinders, a rough layer, and an object below a rough surface," Journal of the Optical Society of America A: Optics and Image Science, and Vision, vol. 30, no. 9, pp. 17271737, 2013.

[9] R.-W. Xu, L.-X. Guo, and R. Wang, "An iterative analytic Numerical method for scattering from a target buried beneath a rough surface," Chinese Physics B, vol. 23, no. 11, Article ID 114101, 2014.

[10] S. Bellez, C. Bourlier, and G. Kubické, “3-D scattering from a PEC target buried beneath a dielectric rough surface: an efficient PILE-ACA algorithm for solving a hybrid KA-EFIE formulation," Institute of Electrical and Electronics Engineers. Transactions on Antennas and Propagation, vol. 63, no. 11, pp. 5003-5014, 2015.

[11] X. Millard and Q. H. Liu, "A fast volume integral equation solver for electromagnetic scattering from large inhomogeneous objects in planarly layered media," Institute of Electrical and Electronics Engineers. Transactions on Antennas and Propagation, vol. 51, no. 9, pp. 2393-2401, 2003.

[12] Y. Ren, Q. H. Liu, and Y. P. Chen, "A hybrid FEM/MoM Method for 3-D electromagnetic scattering in layered medium," Institute of Electrical and Electronics Engineers. Transactions on Antennas and Propagation, vol. 64, no. 8, pp. 3487-3495, 2016.

[13] R.-W. Xu, L.-X. Guo, H.-J. He, and W. Liu, "A Hybrid FEM/MoM Technique for 3-D Electromagnetic Scattering from a Dielectric Object above a Conductive Rough Surface," IEEE Geoscience and Remote Sensing Letters, vol. 13, no. 3, pp. 314-318, 2016.

[14] H.-J. He and L.-X. Guo, "A Multihybrid FE-BI-KA Technique for 3-D Electromagnetic Scattering from a Coated Object above a Conductive Rough Surface," IEEE Geoscience and Remote Sensing Letters, vol. 13, no. 12, pp. 2009-2013, 2016.

[15] H. Ye and Y.-Q. Jin, "Parameterization of the tapered incident wave for numerical simulation of electromagnetic scattering from rough surface," IEEE Transactions on Antennas and Propagation, vol. 53, no. 3, pp. 1234-1237, 2005.

[16] J. M. Jin, The Finite Element Method in Electromagnetics, Wiley, New York, NY, USA, 1993.

[17] L. Tsang, T. H. Kong, and K. H. Ding, Scattering of Electromagnetic Waves: Numerical Simulations, Wiley, New York, NY, USA, 2001. 


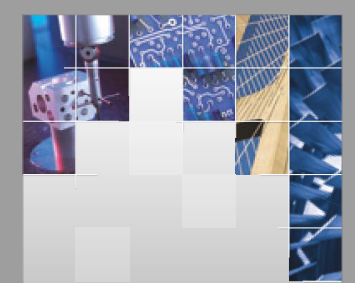

\section{Enfincering}
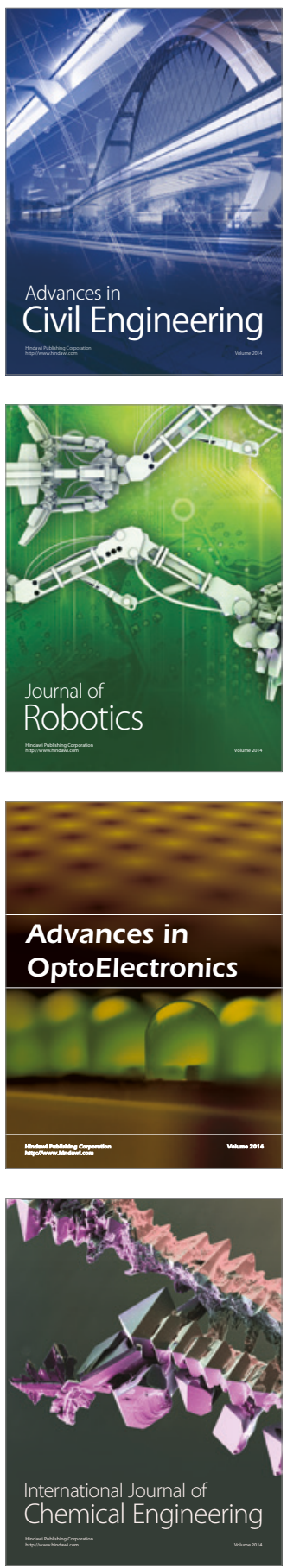

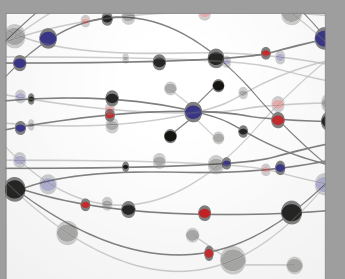

The Scientific World Journal

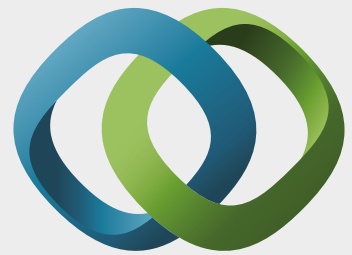

\section{Hindawi}

Submit your manuscripts at

https://www.hindawi.com
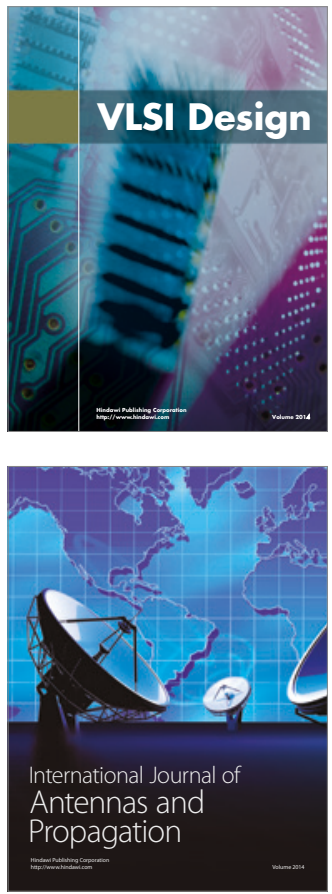

\section{Rotating}

Machinery
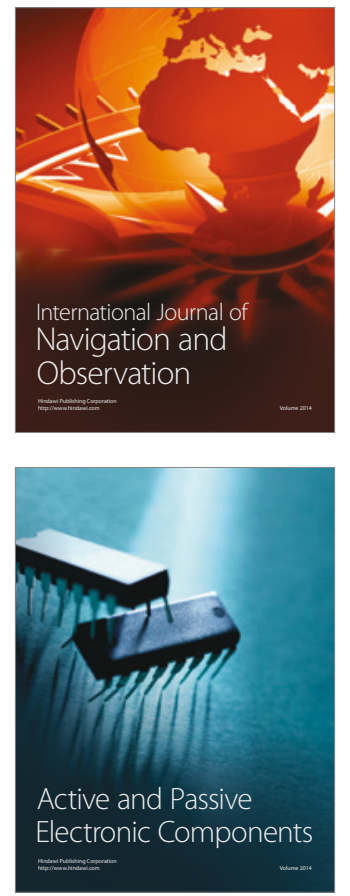
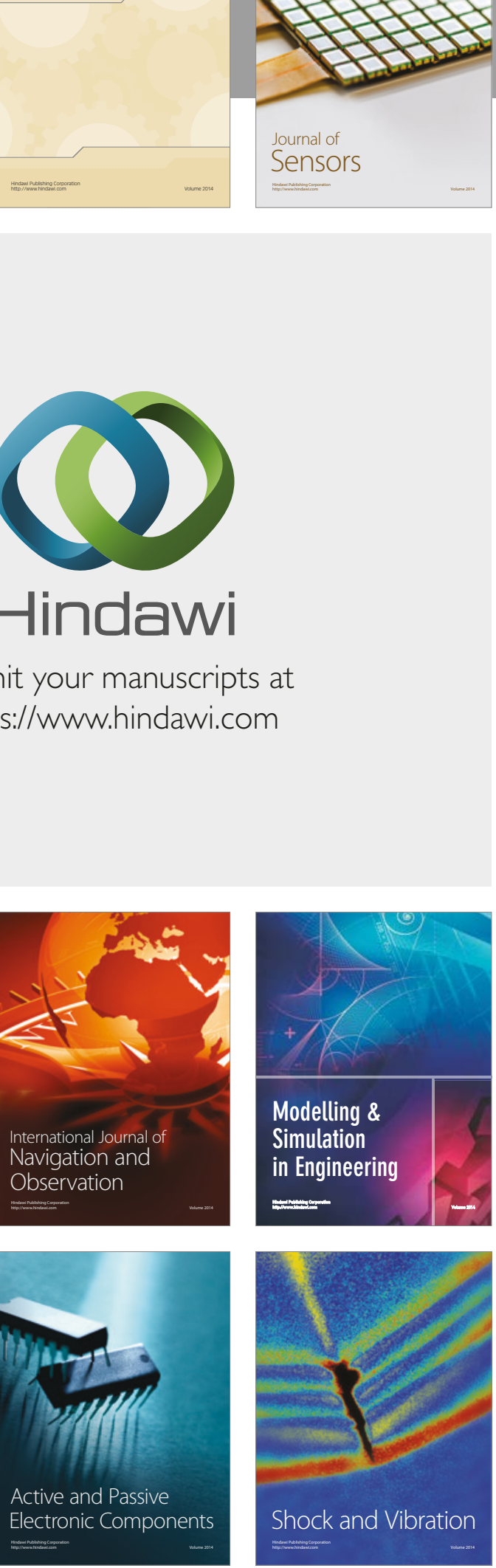
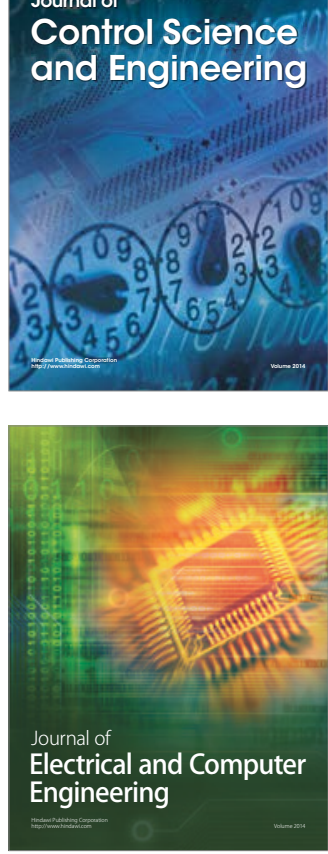

Distributed

Journal of

Control Science

and Engineering
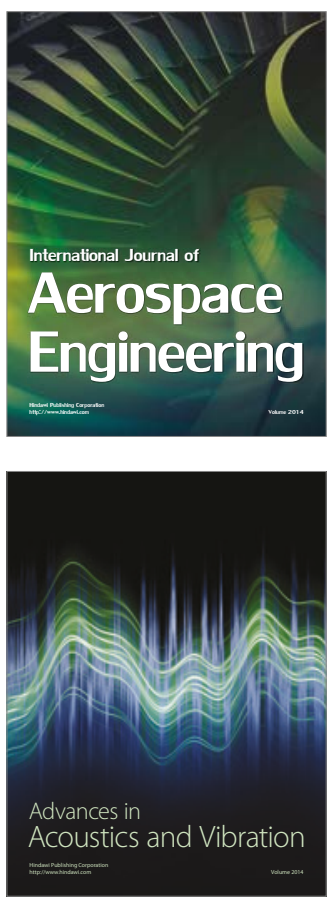

Sensor Networks 